\title{
Efficient parametric amplification in high and very high frequency piezoelectric nanoelectromechanical systems
}

\author{
R. B. Karabalin, ${ }^{1}$ S. C. Masmanidis, ${ }^{1,2}$ and M. L. Roukes ${ }^{1, a)}$ \\ ${ }^{1}$ Kavli Nanoscience Institute, California Institute of Technology, Pasadena, California 91125, USA \\ ${ }^{2}$ Broad Fellows Program in Brain Circuitry, California Institute of Technology, Pasadena, California 91125, \\ USA
}

(Received 28 July 2010; accepted 29 September 2010; published online 1 November 2010)

\begin{abstract}
Parametric amplification in nanomechanical structures is demonstrated by modulating a purely intrinsic mechanical parameter of the system-the stress-via piezoelectric electromechanical coupling. Large resonance amplitude and quality factor enhancement due to parametric pumping are observed under both vacuum and ambient pressure conditions. Exploration of the region of parametric instability yields results that agree with parametric amplification theory. (C) 2010 American Institute of Physics. [doi:10.1063/1.3505500]
\end{abstract}

Recent technological advances have enabled the fabrication of mechanical resonators down to the nanometer scale. ${ }^{1}$ Resonant nanoelectromechanical systems (NEMS) possess such properties as extremely high frequencies, ${ }^{2,3}$ high mechanical responsivity, large quality factors, and operability at low required power, making NEMS resonators promising in a variety of applications ranging from mass and force sensing ${ }^{4-6}$ to quantum limited measurements. ${ }^{7}$ However, as electromechanical systems are miniaturized, readout of mechanical motion becomes increasingly difficult, as most forms of electrical transduction signals scale with device size. One potential solution is amplification of the signal in the mechanical domain using parametric effect before applying electrical transduction.

Parametric resonance is of interest to many areas of research ranging from quantum optics ${ }^{8}$ to plasma physics. ${ }^{9}$ Since the initial demonstration of a resonant mechanical parametric system by Rugar and Grütter, ${ }^{10}$ it has attracted significant attention. ${ }^{11-14}$ The simplest parametric amplification scheme involves periodic modulation of the spring constant of the system at twice its fundamental resonance frequency. In previous manifestations of mechanical parametric amplification, the nature of the spring constant modulation was usually capacitive, ${ }^{11,12}$ although other methods exploited intrinsic residual stress, ${ }^{14}$ Lorentz force, ${ }^{15}$ or an external feedback loop. ${ }^{16}$ Direct parametric tuning of the tensile stress using piezoelectric electromechanical coupling was introduced earlier in large scale microelectromechanical resonator operating at $140 \mathrm{kHz} .{ }^{17}$ Here, we demonstrate piezoelectric parametric amplification and actuation in high frequency and very-high frequency, submicron-scale NEMS resonators.

Modulation of the stress of a nanomechanical doubly clamped beam results in resonance frequency change: ${ }^{18}$

$$
f=\left(1.03 \frac{t_{h}}{L^{2}} \sqrt{\frac{E}{\rho}}\right) \sqrt{1+\frac{\sigma L^{2}}{3.4 E t_{h}^{2}}},
$$

for length $L$, Young's modulus $E$, density $\rho$, dimension in the direction of vibration $t_{h}$, and stress $\sigma$. The displacement of the central point of the beam is modeled as harmonic oscillator of effective mass $m$, spring constant $k_{1}$, and quality factor $Q$ with Duffing ${ }^{19}$ nonlinearity we obtain the following equation of motion:

${ }^{a)}$ Electronic mail: roukes@caltech.edu.

$$
m \ddot{x}+\frac{m \omega_{0}}{Q} \dot{x}+\left[k_{1}+k_{p} \cos \left(2 \omega_{0} t\right)\right] x+k_{3} x^{3}=F(t),
$$

where $k_{p}=0.3 k_{1} L^{2} \sigma /\left(E t_{h}^{2}\right)$ is the spring constant modulation amplitude, and $F(t)=F_{0} \cos \left(\omega_{D} t+\phi\right)$ is a driving force. The parametric modulation is performed at twice the resonance frequency, leading to a vibrational amplitude gain given by: ${ }^{10}$

$$
G=\frac{A_{\text {pump on }}}{A_{\text {pump off }}}=\left[\frac{\cos ^{2}(\varphi+\pi / 4)}{\left(1+k_{p} / k_{t}\right)^{2}}+\frac{\sin ^{2}(\varphi+\pi / 4)}{\left(1-k_{p} / k_{t}\right)^{2}}\right]^{1 / 2},
$$

where $k_{t}$ is the threshold parametric pumping force $2 k_{1} / Q$. The second term in this equation diverges as $k_{p}$ approaches $k_{t}$, thus significant vibrational amplitude gain can be obtained, provided the appropriate phase relationship between the parametric modulation and driving force.

Besides the amplitude increase, parametric amplification results in what is, effectively, a quality factor enhancement, which can be observed in drive frequency $\omega_{D}=\omega_{0}+\Delta \omega_{D}$ sweep measurements. As the pumping force is increased, the resonance peak becomes substantially taller and narrower and assumes a non-Lorentzian form described by ${ }^{20}$

$$
A=\frac{F_{0} Q}{m \omega_{0}^{2}} \frac{2 Q \Delta \omega_{D}-i \omega_{0}}{\omega_{0}\left(1-k_{p}^{2} / k_{t}^{2}\right)-4 Q^{2} \Delta \omega_{D}^{2} / \omega_{0}+4 i Q \Delta \omega_{D}} .
$$

Piezoelectricity is a well-known phenomenon that directly converts electric field into mechanical strain. The devices are fabricated from epitaxially grown of GaAs p-i-n diodes: $100 \mathrm{~nm}$ highly $\mathrm{n}$-doped, $50 \mathrm{~nm}$ insulating and another $50 \mathrm{~nm}$ layer of highly p-doped GaAs. It has been shown in previous work ${ }^{2 P}$ that these structures enable efficient actuation as well as a frequency tuning.. We fabricated six doubly clamped beams on the same chip with lengths ranging from 3 to $8 \mu \mathrm{m}$, and a width of $500 \mathrm{~nm}$ [Fig. 1(a)]. Displacement measurements were carried out in vacuum ( $\sim 10 \mathrm{mTorr})$ and room temperature using optical interferometry. ${ }^{3,22}$ The fundamental frequencies range from 11 to $53 \mathrm{MHz}$. The quality factor varies from 2650 for the longest beam down to 1600 for the shortest beam.

Each device's resonance frequency is precisely tracked as a dc bias between the top and bottom conducting layers is varied. The results of these measurements are shown in Fig. 1(b). All devices exhibit approximately equal frequency shifts corresponding to $\sim 40 \mathrm{kHz} / \mathrm{V}$. When a dc bias is applied, the anisotropic piezoelectric coupling of the material 


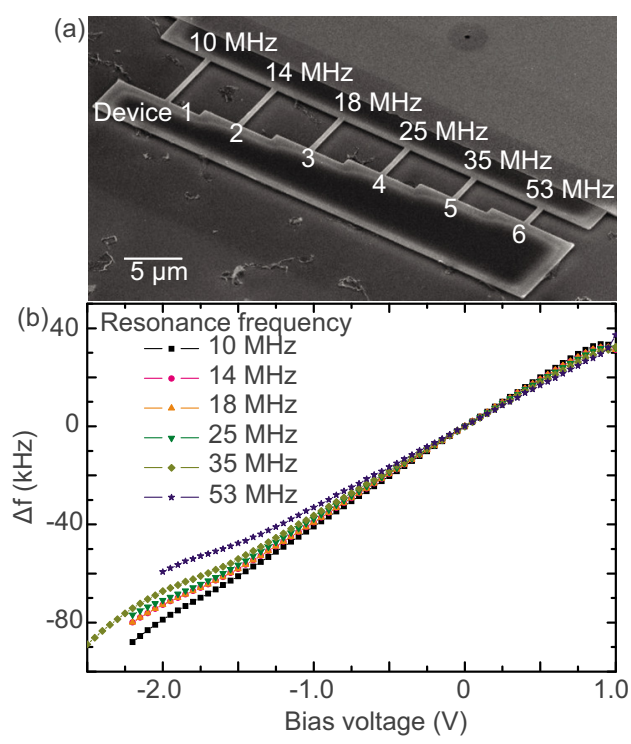

FIG. 1. (Color online) (a) SEM image of the series of parametric resonators and amplifiers. The geometrical parameters of the beams are $3<L$ $<8 \mu \mathrm{m}, w=500 \mathrm{~nm}$, and $t=200 \mathrm{~nm}$. (b) The piezoelectric frequency tuning of the resonators obtained by dc biasing the beams. The linear dependence of the frequency shift closely matches the predictions from piezoelectric theory.

causes longitudinal strain to develop in the intermediate insulating layer. Doubly clamped boundary conditions convert the strain to stress, which either stiffens or softens the beam, thereby changing its resonance frequency. ${ }^{21,22}$ We thus demonstrate that tensile stress modulation via this piezoelectric coupling approach is characterized by exceptional linearity across a wide frequency range. This is in contrast to the Lorentz-force based parametric modulation we have previously reported, where the excess Joule heating compromised the linearity of frequency modulation. ${ }^{15}$

Due to the efficiency of the piezoelectric stress tuning mechanism, the parametric resonance can be implemented at very high frequencies. When we parametrically pump the resonator at twice the characteristic frequency of device no. 6 (the shortest beam in Fig. 1(a); $L=3 \mu \mathrm{m} f_{0}=53.3 \mathrm{MHz}$ ), quality factor enhancement is observed via frequency scanned measurements. The inset of Fig. 2(a) displays a set of parametrically modified resonance peaks under different pumping voltages but constant driving voltage. Effective quality factors of 119000 are observed as pump voltages approach the threshold. The modified frequency response closely matches theoretical predictions given by Eq. (4). The curves in Fig. 2(a) represent the amplitude gain under three representative drive voltages, and show that small signal gains of 200 can be achieved for 53.3 MHz NEMS parametric amplifier.

Parametric amplification is particularly promising for nanomechanical resonators operating in ambient environments. ${ }^{16,23}$ We demonstrate this by carrying out similar experiments at atmospheric pressure. The results from the longest available beam (device no. $1 \quad L=8 \mu \mathrm{m} \quad f_{0}$ $=10.9 \mathrm{MHz})$ are displayed in Fig. 2(b). Direct parametric gain measurements yielded a thousand-fold amplitude increase. As shown in the inset of Fig. 2(b), the parametric resonator has a damped $Q$ of 130 in air but achieves a $Q$ effectively enhanced to a value of 4900 near the threshold.
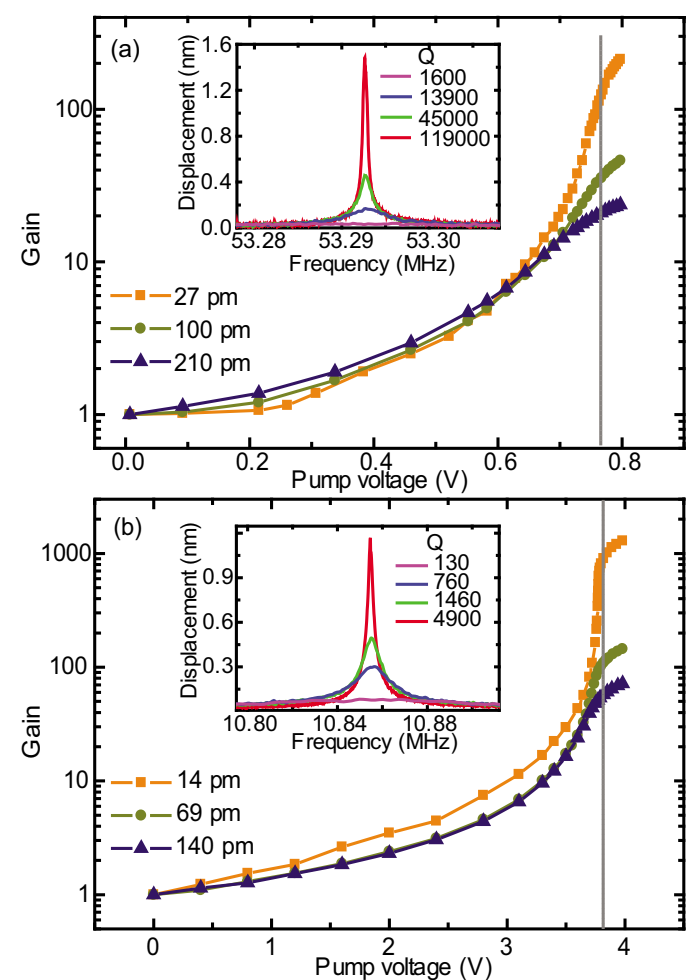

FIG. 2. (Color online) (a) The dependence of gain on pump amplitude for different initial drives for the device no. $6\left(f_{0}=53.3 \mathrm{MHz}, L=3 \mu \mathrm{m}, w\right.$ $=500 \mathrm{~nm}, t=200 \mathrm{~nm}$ ) in vacuum. A small-signal gain of 200 is observed. Inset: effective quality factors of the parametrically enhanced resonance peaks are 1600 with no pump,13 900 and 45000 with intermediate pump and 119000 close to threshold. (b) The dependence of gain on pumping voltage for different initial drives for the device no. $1(L=8 \mu \mathrm{m}, w$ $=500 \mathrm{~nm}, t=200 \mathrm{~nm})$ in ambient conditions. A small-signal gain of 1000 is observed. Inset: effective quality factors of the parametrically enhanced resonance peaks are 130 with no pump, 760 and 1460 with intermediate pump, and 4900 close to the threshold.

Up to now we have primarily explored the subthreshold regime. When the pumping force exceeds threshold, the amount of energy delivered into the system is larger than the amount lost to dissipation. In this situation, in the absence of any driving signal, the resonator executes spontaneous oscillations close to the resonance frequency. The intricate behavior of this dynamical system is described in detail in Refs. 11 and 13. An important characteristic of parametric resonance is the presence of an instability region in pump amplitude and frequency space, known asparametric, "tongue." Its lowest point corresponds to the threshold condition described in Eq. (3), given by a parametric frequency of $2 \omega_{0}$ and a pump level of $2 k_{1} / Q$.

All of the devices shown in Fig. 1(a) have instability thresholds that are experimentally accessible. In this work most of the characterizations are performed with device no. 5 $\left(L=4 \mu \mathrm{m}, f_{0}=35 \mathrm{MHz}\right)$. Instability threshold measurements were carried out by removing the direct drive source, leaving only the parametric pump. The output signal from the photodetector is then measured with a spectrum analyzer.

Figure 3(a) shows examples of upward and downward parametric sweeps. During the upward frequency sweep a nontrivial solution becomes the only stable one at the left edge of the instability region (around $35.2876 \mathrm{MHz}$ ); as a result the beam starts oscillating. With increasing pump frequency the amplitude of mechanical motion increases with $\sim \omega^{1 / 2}$ dependence, in agreement with theoretical predictions. 

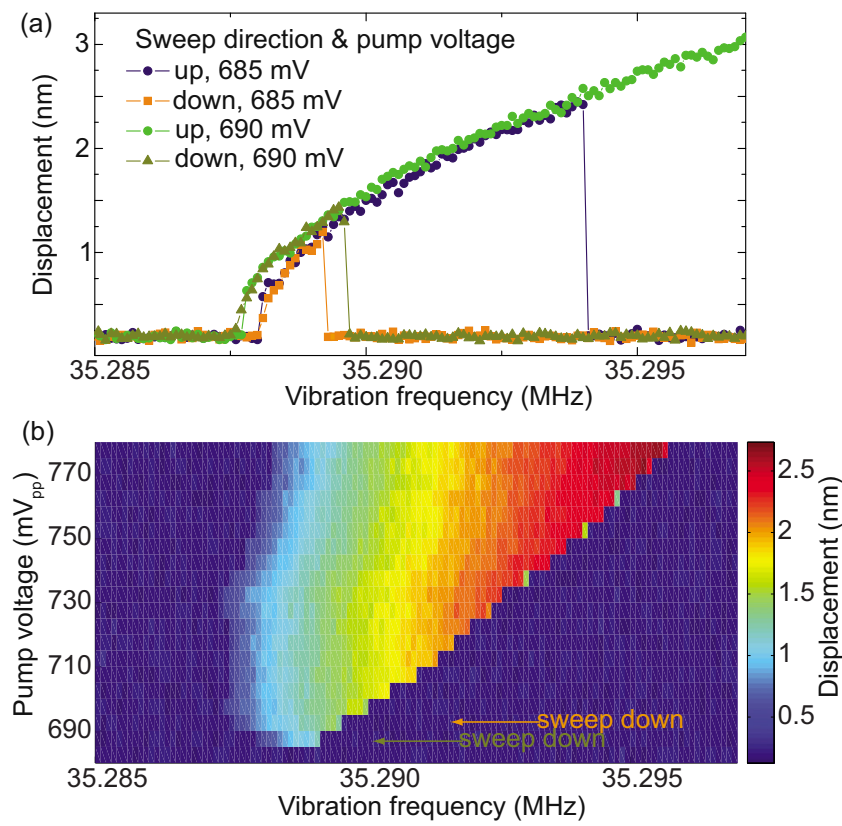

FIG. 3. (Color online) (a) An example of upward and downward parametric frequency sweeps. The onset of instability and regions of bistability are clearly visible. (b) The parametric tongue is mapped by plotting the set of downward sweeps at different pump voltages. The amplitude of mechanical motion is color-coded.

When the pump frequency reaches the right edge of the tongue, the mechanical motion does not cease, because both trivial and nontrivial solutions are stable. Hence the amplitude continues to increase until other factors such as nonlinear viscous damping ${ }^{13}$ cause the motion to stop. On the other hand, during the downward sweep, the mechanical motion jumps to high amplitude at the right edge of the tongue (35.2889 MHz). Later the amplitude of motion decreases to zero along the same curve as followed on the upward sweep, as shown in Fig. 3(a).

According to theoretical analysis, ${ }^{11}$ the instability region widens as the pump increases. The experimental measurements shown in Fig. 3(b) confirm this prediction. The collection of downward sweeps with steadily increased pump provides a map of the parametric tongue. The $x$-axis in Fig. 3(b) is a frequency of mechanical motion (half pump frequency), the $y$-axis is pump amplitude in millivolts peak-to-peak $\left(\mathrm{mV}_{\mathrm{pp}}\right)$ and the amplitude of mechanical motion signal is represented by color scale. We note that the predicted parametric threshold calculated from quality factor and tunability measurements shown in Fig. 1(b), is $678 \mathrm{mV}_{\mathrm{pp}}$, which matches the measured value.
Mechanical parametric amplification is a valuable effect that can benefit a large variety of NEMS applications. Here, we have demonstrated an efficient scheme for efficient frequency tuning in high frequency NEMS. Thousand-fold amplitude gain and 75-fold effective quality factor enhancement were experimentally measured. Such phenomena could be applied as amplifiers operating in the mechanical domain before applying conventional electronics, aiding transduction of nanomechanical motion.

We thank Mike Cross and Matt Matheny (Caltech) and Ron Lifshitz (Tel Aviv University) for many useful discussions and suggestions. We also thank Iwijn De Vlaminck and Gustaaf Borghs (IMEC, Leuven, Belgium) for providing us with the GaAs material used in this work.

${ }^{1}$ M. L. Roukes, Phys. World 14, 25 (2001).

${ }^{2}$ X. M. H. Huang, C. Zorman, M. Mehregany, and M. L. Roukes, Nature (London) 421, 496 (2003).

${ }^{3}$ N. Liu, F. Giesen, M. Belov, J. Losby, J. Moroz, A. E. Fraser, G. Mckinnon, T. J. Clement, V. Sauer, W. K. Hiebert, and M. R. Freeman, Nat. Nanotechnol. 3, 715 (2008).

${ }^{4}$ Y. T. Yang, C. Calegari, X. L. Feng, K. L. Ekinci, and M. L. Roukes, Nano Lett. 6, 583 (2006).

${ }^{5}$ K. Jensen, K. Kim, and A. Zettl, Nat. Nanotechnol. 3, 533 (2008).

${ }^{6}$ D. Rugar, R. Budakian, H. J. Mamin, and B. W. Chui, Nature (London) 430, 329 (2004).

${ }^{7}$ M. D. LaHaye, O. Buu, B. Camarota, and K. C. Schwab, Science 304, 74 (2004).

${ }^{8}$ L. Wu, H. J. Kimble, J. L. Hall, and H. Wu, Phys. Rev. Lett. 57, 20 (1986).

${ }^{9}$ E. Esarey, P. Sprangle, J. Krall, and A. Ting, IEEE Trans. Plasma Sci. 24, 252 (1996).

${ }^{10}$ D. Rugar and P. Grütter, Phys. Rev. Lett. 67, 699 (1991).

${ }^{11}$ K. L. Turner, S. A. Miller, P. G. Hartwell, N. C. MacDonald, S. H. Strogatz, and S. G. Adams, Nature (London) 396, 149 (1998).

${ }^{12}$ D. W. Carr, S. Evoy, L. Sekaric, H. G. Craighead, and J. M. Parpia, Appl. Phys. Lett. 77, 1545 (2000).

${ }^{13}$ R. Lifshitz and M. Cross, Phys. Rev. B 67, 134302 (2003).

${ }^{14}$ A. Dâna, F. Ho, and Y. Yamamoto, Appl. Phys. Lett. 72, 1152 (1998).

${ }^{15}$ R. B. Karabalin, X. L. Feng, and M. L. Roukes, Nano Lett. 9, 3116 (2009).

${ }^{16}$ M. Moreno-Moreno, A. Raman, J. Gomez-Herrero, and R. Reifenberger, Appl. Phys. Lett. 88, 193108 (2006).

${ }^{17}$ I. Mahboob and H. Yamaguchi, Appl. Phys. Lett. 92, 253109 (2008).

${ }^{18} \mathrm{~S}$. Timoshenko, D. H. Young, and W. Weaver, Jr., Vibration Problems in Engineering (Wiley, New York, 1974).

${ }^{19}$ A. H. Nayfeh and D. T. Mook, Nonlinear Oscillations (Wiley, New York, 1979).

${ }^{20} \mathrm{R}$. Lifshitz and M. C. Cross, Reviews of Nonlinear Dynamics and Complexity, edited by H. G. Shuster (Wiley, New York, 2008), Vol. 1, p. 52.

${ }^{21}$ S. C. Masmanidis, R. B. Karabalin, I. De Vlaminck, G. Borghs, M. R. Freeman, and M. L. Roukes, Science 317, 780 (2007).

${ }^{22}$ R. B. Karabalin, M. H. Matheny, X. L. Feng, E. Defaÿ, G. L. Rhun, C. Marcoux, S. Hentz, P. Andreucci, and M. L. Roukes, Appl. Phys. Lett. 95, 103111 (2009).

${ }^{23}$ W. Zhang and K. L. Turner, Sens. Actuators, A 122, 301 (2005). 